\title{
Decomposing the Socioeconomic Inequality in Delivery Care in Egypt
}

\author{
Mohamed Ali Hussein Aboakrab, Ph.D. \\ Lecturer, Statistics Department, Faculty of Politics and \\ Economics, Beni-Suef University, Egypt
}

\begin{abstract}
:
Health care effectiveness has numerous indicators to measure, but women death during pregnancy, delivery, or postpartum period is one of the most important measures in any country. Access to skilled attendance during childbirth will contribute to reduce in maternal mortality. Disparities are existing inside and among countries in use of reproductive health services. This paper aims to measure the level of inequalities in use of delivery health services and to clarify the determinants of inequalities in Egypt. The data utilized were obtained from the survey of demographic and health in Egypt. The outcome variables of maternal health care were skilled assistance during childbirth and delivery at a health facility. The findings revealed that utilization of delivery health care was concentrated among rich and educated mothers. In general, the variable economic status was seen as the most significant factor sharing in the inequality in use of delivery services in Egypt, alongside women's education.
\end{abstract}

Keywords: Decomposing, Inequality, Delivery care, Health facility, Skilled birth attendance, Socioeconomic, Egypt 


\section{Introduction}

The estimated 2017 global maternal mortality ratio (MMR) is 211 deaths for each 100000 women giving birth. The overall proportion of women's deaths that are because of maternal causes was evaluated at 9.2 percent in 2017. The Sustainable Development Goals (SDGs) assure healthy lives for all at all ages through the third goal. The SDG target 3.1 intends to decrease the global MMR to under 70 deaths for each 100000 women giving birth by 2030 . Achieving this global target will save around 1.4 million mothers' lives from 2016 to 2030. It is necessary for countries to reduce their MMR by 6.1 percent every year from 2016 to 2030 to achieve the SDG target 3.1 (WHO, 2019).

There are disparities among countries in maternal mortality ratio. About 66 percent of the estimated universal number of maternal deaths in 2017 was in Sub-Saharan Africa. The nationallevel data often show inequalities in MMR that exist between population groups within the countries. These disparities are across age groups and education levels. The SDG 10 goal intends to decrease inequality inside and among countries. Address inequities in utilization of maternal care and ensure common health coverage for maternal care are part of the strategic framework for ending preventable maternal mortality (WHO, 2019).

The current paper is an attempt to find out the causes' contributions of socioeconomic inequality in delivery health care utilization in Egypt by using Wagstaff decomposition. 


\section{Research Problem}

The MMR in Egypt was 37 deaths per 100,000 women giving birth in 2017. The average annual rate of decrease in MMR from 2000 to 2017 is 3.2 percent (WHO, 2019). One of the reasons of maternal mortality is childbirth (WHO, 2005). Skilled birth assistance may help to reduce maternal mortality (WHO, 2014).

Our previous study has described degree of the socio-economic inequality in national and subnational level for three indicators of maternal health: skilled assistance during childbirth, delivery at a health facility and postnatal care utilization in Egypt utlizing data from EDHS 2014. The study used concentration index and concentration curve to assess inequality degree (Hussein, 2018). Our current paper attempts to contribute to the extant literature through using Wagstaff decomposition to indicate the relative share of socioeconomic determinants to inequalities in utilization of delivery care.

\section{Review of Literatures}

Houweling et al. (2007) aimed to monitor inequalities in skilled assistance during childbirth for 43 countries. Data used for this paper were taken from the DHS in these countries. The study used absolute measures (rate difference) and relative measures (rate ratio) to examine the inequalities. The findings showed that mothers from higher socioeconomic groups are more likely to utilize delivery health care services.

Khadr (2009) studied the progress in reproductive health indicators in Egypt using three rounds of the EDHS (1995, 2000 and 2005). The study used concentration index to examine inequalities in reproductive health indicators: childbearing, early 
marriage, fertility, contraceptive use, delivery, prenatal and postnatal checkup. It was reported in this literature that contraceptive use and fertility demonstrated decreases in inequalities. Moreover, other indicators such as delivery and antenatal care showed high degrees of inequality.

Zere et al. (2011) decomposed inequalities in skilled attendance during childbirth in Namibia. Data were taken from the Namibia DHS 2006. The findings of the concentration index and curve indicated significant inequalities in presence of a skilled health person during childbirth. The findings revealed that inequalities in wealth and women's education are demonstrated the most important determinants of inequities in delivery care by skilled provider.

Liu et al. (2014) clarified socioeconomic inequalities in reproductive health services using decomposition of the concentration index in China. Data used for this study were derived from 10 provinces in China in 2005. The findings of the decomposition analysis showed that the greater part of the inequality in use of reproductive health care services may be interpreted by disparities in women's age, wealth status, education of parents and ethnicity.

Bobo et al. (2017) measured inequities in use of reproductive health care dependent on four determinants: urban/rural, region, education of mother and wealth index. This prior study addressed inequities in utilization of delivery in health facility, antenatal health care, postnatal checkup and contraceptive methods using data from Ethiopian DHS 2014. The paper used the concentration curve, the equity gaps, the concentration index, and the rate-ratios to achieve its objective. The results of this study indicated that inequities in health care utilization were shown favoring mothers in urban residents, developed regions, most educated and the wealthy. 
Hussein (2018) examined level of the socio-economic disparities in reproductive health services in national and subnational level in Egypt. He studied three indicators of maternal health: skilled assistance during childbirth, delivery at a health facility and postnatal care utilization using data from EDHS 2014. This study measured inequality using concentration curve, simple measures (difference and ratio) and complex measures (concentration index). The findings of this previous study indicated that usage of facility delivery, skilled assistance during childbirth and postnatal care were more concentrated among the richest and the most educated mothers at national and subnational regions.

Huda et al. (2018) investigated the determinants of inequalities in use of facility delivery services in Bangladesh, Pakistan and Nepal using decomposition of concentration index. Data were taken from DHS of Nepal 2010, Pakistan 2012, and Bangladesh 2014. The findings of the decomposition method showed that wealth status, education of parents, parity, and urban/rural were the most significant determinants of inequalities in use of facility delivery services.

Novignon et al. (2019) measured socioeconomic inequalities in delivery by trained attendants in Ghana using decomposition of the concentration index. Data used for this study were taken from the Ghana DHS (2003, 2008 and 2014). The results of the decomposition method demonstrated that the most important determinants of inequality in delivery by trained attendants were women's education and access to health insurance.

To sum up, previous studies clarified the inequality in indicators of delivery services in Egypt using only the concentration index (Hussein 2018; 11]. Our current paper utilizes Wagstaff decomposition to know contributions of socioeconomic inequality in delivery services utilization in Egypt. 


\section{Data Source}

The present study used the most recent dataset available in Egypt in 2014. This data were from 2014 Egypt Demographic and Health Survey (EDHS). El-Zanaty made this survey in Egypt on interest of the Ministry of Health. From samples of 21,762 ever married women aged 15 - 49, this current study included just women whose last birth was during the five-year period before the survey. They amounted to 11,391 mothers.

\section{Methodology}

Descriptive statistics are carried out to estimate the prevalence of delivery services utilization among women by different demographic and socioeconomic factors. Moreover, Chi-square test is utilized to distinguish the significant association between the utilization of delivery care services and some selected socioeconomic characteristics.

Turning to the decomposition analysis, two binary maternal healthcare indicators were examined. The first variable is whether a woman reported giving birth at a health facility (public or private). The second variable is whether a woman reported receiving skilled assistance during childbirth. The factors potentially sharing of inequality in delivery care that were included in this paper were wealth index, husband's education, mother's education, mass media (listening to Radio - watching TV- reading newspaper), having a terminated pregnancy, work status, woman's age, place of residence and region.

Measuring the socioeconomic inequality in delivery care was performed using two steps. The first step was calculating the concentration index to measure the amount of inequality. The second step was calculating the share of every independent variable to the inequality in delivery care by decomposition method. 
The concentration index (C) with range lying between -1 and 1 is calculated using the covariance between the use of delivery care and the fractional rank of the individual sorted by wealth status. It can be written as:

$$
C=\frac{2}{\mu} \sum_{t=1}^{T} f_{t} \mu_{t} R_{t}-1
$$

Where $\mathrm{T}$ is number of groups of socio-economic, $f_{t}$ denotes the proportion of the sample in the group $t, \mu=\sum_{t=1}^{T} f_{t} \mu_{t}$ denotes the overall mean of delivery care, $\mu_{\mathrm{t}}$ is the average value of delivery care amongst the group $\mathrm{t}$, and $R_{t}$ denotes relative rank in the group t (Wagstaff et al., 1991; Wagstaff et al., 1997)..

The method of decomposition proposed by Wagstaff et al. (2003) is a regression method where the concentration index is regressed into its determinants. Moreover, Wagstaff et al. (2003) found that for any linear regression model connecting the dependent variable $\mathrm{y}$ (delivery care), to a set of $\mathrm{k}$ independent variables, $\mathrm{x}_{\mathrm{k}}$ :

$$
y_{i}=\alpha+\sum_{k=1}^{n} \beta_{k} X_{k i}+\varepsilon_{i}
$$

Where $\varepsilon$ denotes an error term. The concentration index $(\mathrm{C})$ for the dependent variable y can be composed as:

$$
C=\sum_{k=1}^{n}\left(\frac{\beta_{k} \bar{x}_{k}}{\mu}\right) C_{k}+\frac{G C_{\varepsilon}}{\mu}
$$

Where $\bar{x}_{k}$ denotes the mean of $\mathrm{x}_{\mathrm{k}} ; \mu$ denotes the mean of $\mathrm{y} ; \mathrm{C}_{\mathrm{k}}$ denotes the concentration index for $\mathrm{x}_{\mathrm{k}} ; \mathrm{GC}_{\varepsilon}$ denotes the generalized concentration index for $\varepsilon_{\mathrm{i}}$. 
The last equation consists of two items. The first is the deterministic component that explained the amount of every factor's share to the measured inequality in delivery care utilization. The second component $\left(\frac{G C_{\varepsilon}}{\mu}\right)$ is the residual that considers the inequality that cannot be clarified by systematic variation in the $\mathrm{x}_{\mathrm{k}}$ across socioeconomic groups.

\section{Results}

\subsection{Descriptive results}

The usage of delivery services according to selected socioeconomic characteristics is shown in Table [1]. About 88 percent of the mothers gave birth in a healthcare facility. Further, around 92 percent of the mothers received skilled attendance during childbirth. It could be noticed that the percentages of women who utilize delivery services increased with education level for woman and her husband, exposure to mass media and wealth index. Regarding women's education, Table [1] shows that delivery at a health facility was about 73 percent for mothers who have never gone to school against 97 percent for highly educated mothers. Meanwhile, about 81 percent of mothers who have never gone to school received skilled attendance during childbirth, while this percentage expanded to about 99 percent for highly educated mothers.

The findings of Table [1] indicate that the poor women were found to have lower delivery care services utilization than the rich women. Delivery at health facility was almost universal among those women from the richest group (about 98 percent) whereas about 76 percent of the poorest women gave their last births in a hospital. About 99 percent of those women from the richest group received skilled attendance during childbirth while the corresponding figure was 83 percent among those from the poorest group. 
Table (1): Utilization of delivery care services by selected socioeconomic characteristics, EDHS 2014

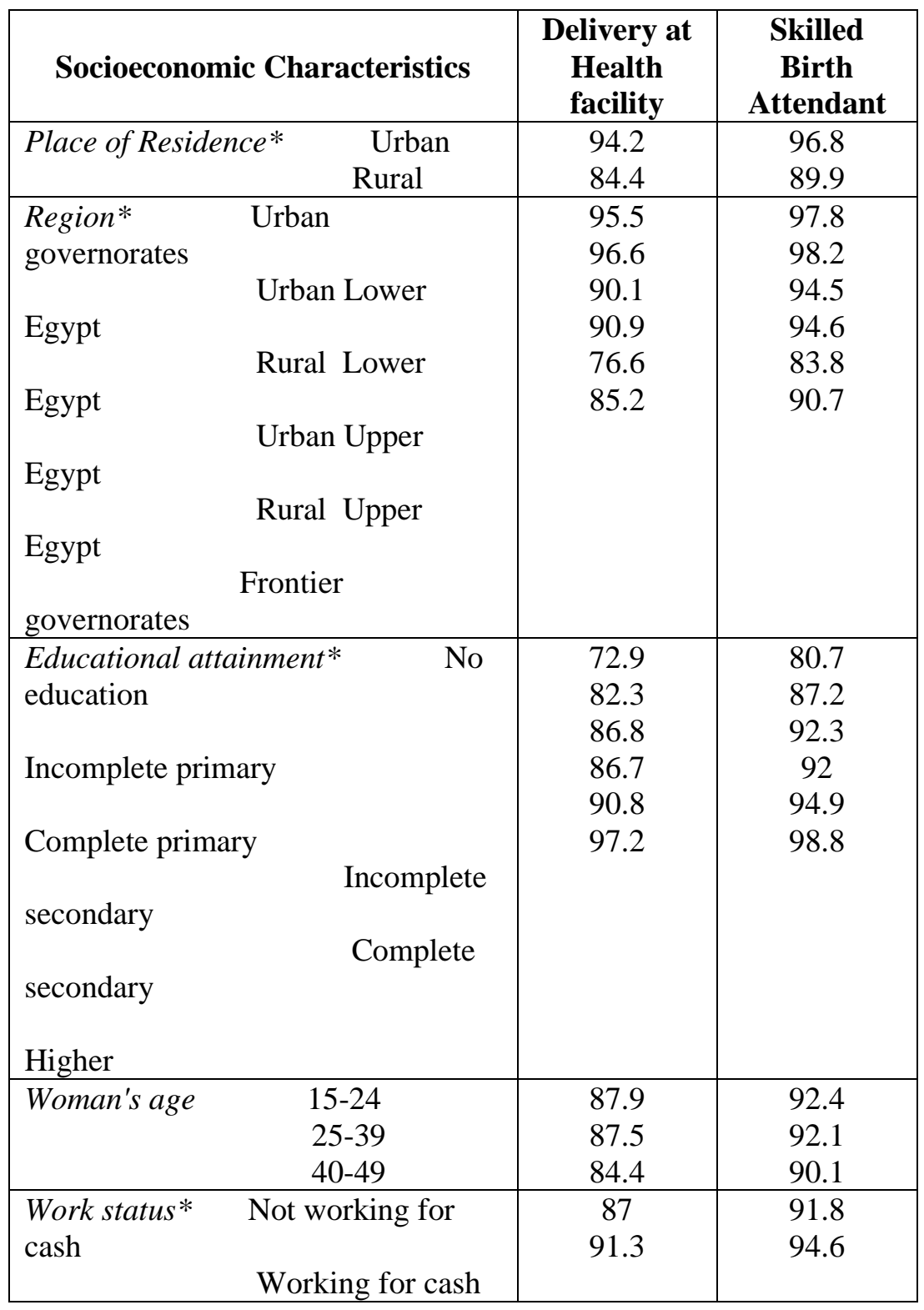




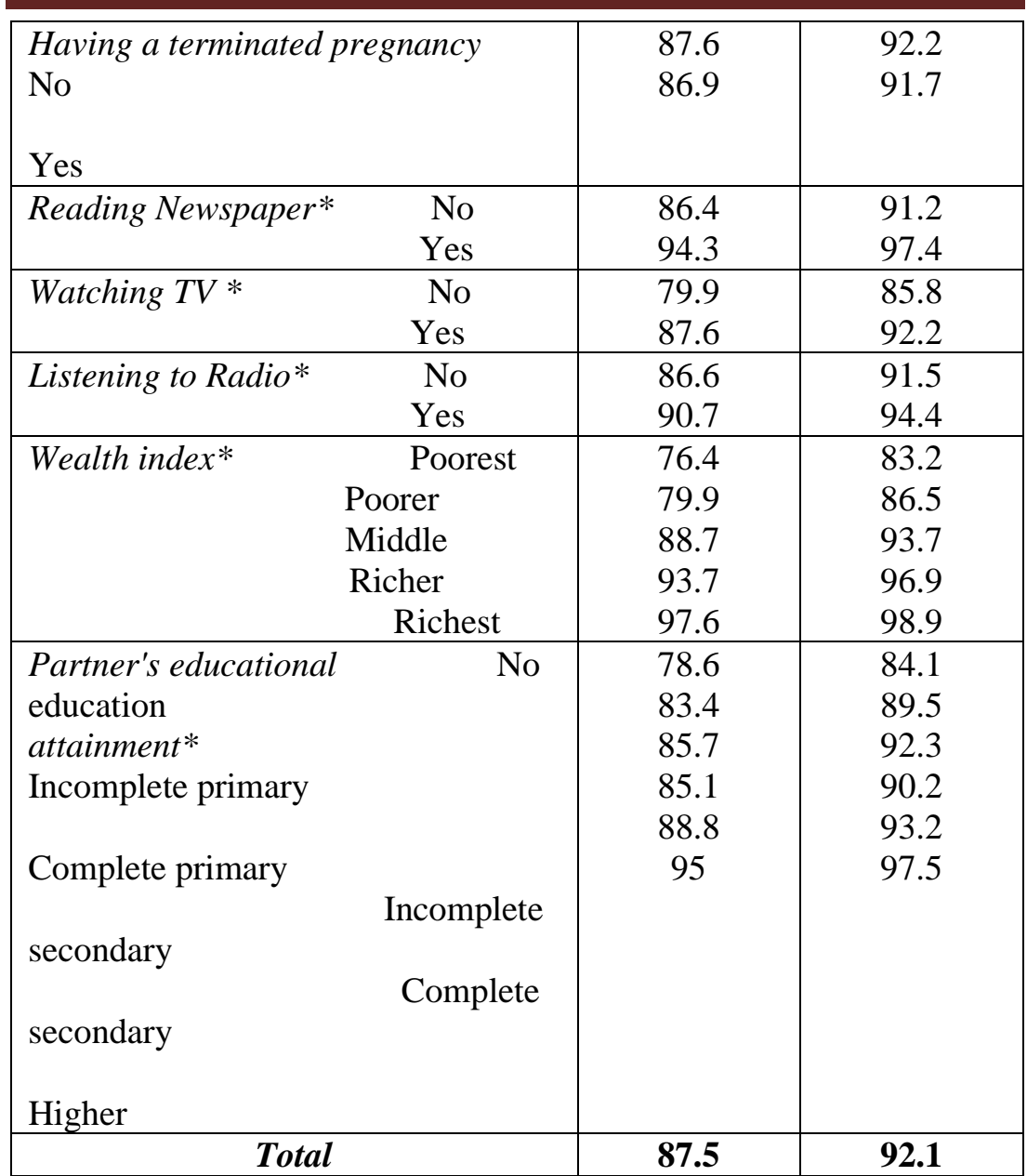

* Significant at $1 \%$

Table [1] indicates that births to women in urban areas were found to have higher delivery care services utilization than those in rural areas. Additionally, mass media exposure significantly increases health facility utilization for childbirth. As shown in Table [1], about 80 percent of mothers who do not watch Television gave their last births in a health facility, while this percentage grew up about 88 percent between mothers who watch Television. Regarding chi-square test, it is demonstrated that there 
is a significant association between utilization of delivery care and education level, mass media exposure, work status, rural/urban, husband`s education, region and wealth index.

\subsection{Decomposition results}

The findings of the determinants to inequalities in use of delivery care are shown in Tables [2] and [3]. The positive values of the concentration indices for skilled assistance during childbirth and institutional delivery indicate that use of delivery services was more concentrated among the mothers belonging to the richest households.

Table [2] shows the decomposition of inequality in delivery at health facility across socioeconomic determinants. It is possible to conclude, from Table [2], that vast majority of the inequality in institutional delivery is clarified by inequalities in households' economic status, mother's education and region. Among these contributions, wealth was the main contributor to the inequality of delivery at health facility. 
Table (2): Decomposing of inequality in delivery at health facility across socioeconomic and demographic variables in

\begin{tabular}{|c|c|c|c|c|c|c|}
\hline \multicolumn{7}{|c|}{ Egypt, EDHS 2014} \\
\hline Variables & $\beta_{k}$ & $\begin{array}{c}\text { Mea } \\
\mathbf{n} \\
\bar{x}_{k}( \\
)\end{array}$ & $\begin{array}{c}\text { Elasticit } \\
\mathbf{y} \\
( \\
\frac{\beta_{k} \bar{x}_{k}}{\mu} \\
\mu \\
{ }^{\prime}\end{array}$ & $C_{k}$ & Share & $\begin{array}{c}\% \\
\text { share }\end{array}$ \\
\hline Residence in urban & $\begin{array}{c}-\overline{-} \\
0.012 \\
3\end{array}$ & $\begin{array}{c}0.318 \\
2\end{array}$ & -0.0045 & $\begin{array}{c}0.566 \\
1 \\
\end{array}$ & $\stackrel{-}{0.0025}$ & -5 \\
\hline \multicolumn{7}{|c|}{ Mother's education (No education ${ }^{\text {a }}$ ) } \\
\hline Incomplete primary* & $\begin{array}{c}0.046 \\
8 \\
\end{array}$ & $\begin{array}{c}0.048 \\
6 \\
\end{array}$ & 0.0026 & $\begin{array}{c}- \\
0.260 \\
6\end{array}$ & $\begin{array}{c}- \\
0.0007\end{array}$ & -1 \\
\hline Complete primary* & $\begin{array}{c}0.056 \\
7\end{array}$ & $\begin{array}{c}0.038 \\
5\end{array}$ & 0.0025 & $\begin{array}{c}- \\
0.117 \\
7 \\
\end{array}$ & $\begin{array}{c}- \\
0.0003 \\
\end{array}$ & -1 \\
\hline Incomplete secondary* & $\begin{array}{c}0.058 \\
8\end{array}$ & $\begin{array}{c}0.143 \\
7\end{array}$ & 0.0097 & $\begin{array}{c}- \\
0.097 \\
7\end{array}$ & $\overline{-}$ & -2 \\
\hline Complete secondary* & $\begin{array}{c}0.083 \\
5 \\
\end{array}$ & $\begin{array}{c}0.430 \\
3 \\
\end{array}$ & 0.0411 & $\begin{array}{c}0.073 \\
9 \\
\end{array}$ & 0.0030 & 6 \\
\hline Higher* & $\begin{array}{c}0.152 \\
9\end{array}$ & $\begin{array}{c}0.160 \\
8 \\
\end{array}$ & 0.0281 & $\begin{array}{c}0.413 \\
4 \\
\end{array}$ & 0.0116 & 24 \\
\hline Sum & & & & & & 26 \\
\hline \multicolumn{7}{|l|}{ Wealth index $\left(\right.$ Poorest $\left.^{a}\right)$} \\
\hline Poorer & $\begin{array}{c}0.005 \\
2 \\
\end{array}$ & $\begin{array}{c}0.193 \\
3 \\
\end{array}$ & 0.0011 & $\begin{array}{c}-\bar{c} \\
0.462 \\
8 \\
\end{array}$ & $\begin{array}{c}- \\
0.0005 \\
\end{array}$ & -1 \\
\hline Middle* & $\begin{array}{c}0.029 \\
7 \\
\end{array}$ & $\begin{array}{c}0.248 \\
5 \\
\end{array}$ & 0.0084 & $\begin{array}{c}- \\
0.021 \\
0\end{array}$ & $\begin{array}{c}- \\
0.0002 \\
\end{array}$ & $\mathbf{0}$ \\
\hline Richer* & $\begin{array}{c}0.065 \\
8\end{array}$ & $\begin{array}{c}0.214 \\
7\end{array}$ & 0.0161 & $\begin{array}{c}0.442 \\
2\end{array}$ & 0.0071 & 15 \\
\hline Richest* & $\begin{array}{c}0.151 \\
0\end{array}$ & $\begin{array}{c}0.171 \\
5 \\
\end{array}$ & 0.0296 & $\begin{array}{c}0.828 \\
5 \\
\end{array}$ & 0.0245 & 50 \\
\hline Sum & & & & & & 63 \\
\hline \multicolumn{7}{|l|}{ Mother's age $\left(15-24^{\mathrm{a}}\right)$} \\
\hline 25-39 & $\begin{array}{c}- \\
0.006 \\
7\end{array}$ & $\begin{array}{c}0.707 \\
1\end{array}$ & -0.0054 & $\begin{array}{c}0.021 \\
6 \\
\end{array}$ & $\stackrel{-}{0.0001}$ & $\mathbf{0}$ \\
\hline 40-49 & $\begin{array}{c}-\overline{-} \\
0.010 \\
8 \\
\end{array}$ & $\begin{array}{c}0.049 \\
7 \\
\end{array}$ & -0.0006 & $\begin{array}{c}- \\
0.057 \\
4 \\
\end{array}$ & $\begin{array}{c}0.0000 \\
4 \\
\end{array}$ & $\mathbf{0}$ \\
\hline Sum & & & & & & $\mathbf{0}$ \\
\hline
\end{tabular}




\begin{tabular}{|c|c|c|c|c|c|c|}
\hline decomposing the socioeconomic & \multicolumn{3}{|c|}{ Mohamed ali Hussein } & \multicolumn{3}{|c|}{ publishing date $24 / 9 / 2020$} \\
\hline \multicolumn{7}{|c|}{ Partner's education (No education ${ }^{a}$ ) } \\
\hline Incomplete primary & $\begin{array}{c}- \\
0.001 \\
2 \\
\end{array}$ & $\begin{array}{c}0.077 \\
1\end{array}$ & -0.0001 & $\begin{array}{c}- \\
0.183 \\
7\end{array}$ & $\begin{array}{c}0.0000 \\
2 \\
\end{array}$ & $\mathbf{0}$ \\
\hline Complete primary & $\begin{array}{c}0.022 \\
2\end{array}$ & $\begin{array}{c}0.057 \\
0\end{array}$ & 0.0015 & $\begin{array}{c}- \\
0.056 \\
2\end{array}$ & $\begin{array}{c}- \\
0.0000 \\
8\end{array}$ & $\mathbf{0}$ \\
\hline Incomplete secondary & $\begin{array}{c}0.001 \\
9\end{array}$ & $\begin{array}{c}0.124 \\
2\end{array}$ & 0.0003 & $\begin{array}{c}- \\
0.091 \\
3\end{array}$ & $\begin{array}{c}- \\
0.0000 \\
2\end{array}$ & $\mathbf{0}$ \\
\hline Complete secondary & $\begin{array}{c}0.005 \\
8\end{array}$ & $\begin{array}{c}0.442 \\
8 \\
\end{array}$ & 0.0029 & $\begin{array}{c}0.014 \\
3 \\
\end{array}$ & $\begin{array}{c}0.0000 \\
4 \\
\end{array}$ & $\mathbf{0}$ \\
\hline Higher & $\begin{array}{c}0.009 \\
0\end{array}$ & $\begin{array}{c}0.170 \\
2\end{array}$ & 0.0018 & $\begin{array}{c}0.339 \\
6 \\
\end{array}$ & $\begin{array}{c}0.0006 \\
0\end{array}$ & 1 \\
\hline Sum & & & & & & 1 \\
\hline \multicolumn{7}{|l|}{$\begin{array}{l}\text { Region (Urban governorates } \\
\text { a) }\end{array}$} \\
\hline Urban Lower Egypt & $\begin{array}{c}0.021 \\
7\end{array}$ & $\begin{array}{c}0.094 \\
0\end{array}$ & 0.0023 & $\begin{array}{c}0.581 \\
2\end{array}$ & 0.0014 & 3 \\
\hline Rural Lower Egypt & $\begin{array}{c}- \\
0.002 \\
4\end{array}$ & $\begin{array}{c}0.389 \\
9\end{array}$ & -0.0011 & $\begin{array}{c}- \\
0.149 \\
2 \\
\end{array}$ & 0.0002 & $\mathbf{0}$ \\
\hline Urban Upper Egypt & $\begin{array}{c}-\overline{-} \\
0.023 \\
6 \\
\end{array}$ & $\begin{array}{c}0.110 \\
9\end{array}$ & -0.0030 & $\begin{array}{c}\mathbf{0 . 4 5 6} \\
\mathbf{0}\end{array}$ & $\begin{array}{c}- \\
0.0014 \\
\end{array}$ & -3 \\
\hline Rural Upper Egypt* & $\begin{array}{c}- \\
0.065 \\
1\end{array}$ & $\begin{array}{c}0.287 \\
7\end{array}$ & -0.0214 & $\begin{array}{c}0.418 \\
5\end{array}$ & 0.0090 & 18 \\
\hline Frontier governorates* & $\begin{array}{c}- \\
0.080 \\
3 \\
\end{array}$ & $\begin{array}{c}0.009 \\
4\end{array}$ & -0.0009 & $\begin{array}{c}0.105 \\
8 \\
\end{array}$ & $\begin{array}{c}- \\
0.0000 \\
9\end{array}$ & $\mathbf{0}$ \\
\hline Sum & & & & & & 18 \\
\hline Working mother & $\begin{array}{c}- \\
0.014 \\
4\end{array}$ & $\begin{array}{c}0.114 \\
2\end{array}$ & -0.0019 & $\begin{array}{c}0.221 \\
5\end{array}$ & $\stackrel{-}{0.0004}$ & -1 \\
\hline $\begin{array}{l}\text { Having a terminated } \\
\text { pregnancy** }\end{array}$ & $\begin{array}{c}0.013 \\
6\end{array}$ & $\begin{array}{c}0.206 \\
1\end{array}$ & 0.0032 & $\begin{array}{c}- \\
0.065 \\
4 \\
\end{array}$ & $\stackrel{-}{0.0002}$ & $\mathbf{0}$ \\
\hline Reading Newspaper & $\begin{array}{c}0.017 \\
6\end{array}$ & $\begin{array}{c}0.139 \\
2\end{array}$ & 0.0028 & $\begin{array}{c}0.284 \\
7 \\
\end{array}$ & 0.0008 & 2 \\
\hline Listening to Radio & $\begin{array}{c}0.001 \\
7\end{array}$ & $\begin{array}{c}0.209 \\
5\end{array}$ & 0.0004 & $\begin{array}{c}0.085 \\
4 \\
\end{array}$ & $\begin{array}{c}0.0000 \\
3 \\
\end{array}$ & $\mathbf{0}$ \\
\hline Watching TV & $\begin{array}{c}0.025 \\
9\end{array}$ & $\begin{array}{c}0.985 \\
1\end{array}$ & 0.0291 & $\begin{array}{c}0.005 \\
4\end{array}$ & 0.0002 & $\mathbf{0}$ \\
\hline Total predicted $\mathrm{C}$ & & & & & $\begin{array}{c}0.0508 \\
2\end{array}$ & 104.15 \\
\hline Residual & & & & & $\begin{array}{c}- \\
0.0020 \\
2\end{array}$ & -4.15 \\
\hline $\begin{array}{l}\text { Total C of delivery at health } \\
\text { facility }\end{array}$ & & & & & $\begin{array}{c}0.0488 \\
3 \\
\end{array}$ & 100 \\
\hline
\end{tabular}


The findings from Table [2] clearly indicate that the highest contribution to socioeconomic inequality in delivery at health facility was due to households' economic status (63 percent) which explained if income was equally divided among households from various socioeconomic groups, then inequality in delivery at health facility would decrease by 63 percent.

One can observe, from Table [2], that the share of mother's education to inequality in delivery at health facility was 26 percent which illustrated if education was equally distributed among different mothers, then inequality in delivery at health facility would decline by 26 percent. Moreover, it should be mentioned that the contribution of region to inequality in health facility utilization at birth was 18 percent.

On the other hand, the findings of the decomposition method for the dependent variable skilled assistance during childbirth are shown in Table [3]. It is possible to conclude, from Table [3], that the variables of wealth index and mother's education could clarify the highest share of skilled birth assistance inequality. The contribution of wealth to inequality in skilled birth assistance was 74 percent which illustrated if the distribution of income was uniformly distributed among households belonging to various socioeconomic groups, then inequality in skilled assistance during childbirth would decline by 74 percent. 
Table (3): Decomposing of inequality in Skilled Birth Assistance across socioeconomic and demographic variables in Egypt,

EDHS 2014

\begin{tabular}{|c|c|c|c|c|c|c|}
\hline Variables & $\beta_{k}$ & $\begin{array}{c}\text { Mea } \\
\mathbf{n} \\
\bar{x}_{k} \\
(\end{array}$ & $\begin{array}{c}\begin{array}{c}\text { Elastici } \\
\text { ty }\end{array} \\
\frac{\beta_{k} \bar{x}_{k}}{\mu} \\
)\end{array}$ & $C_{k}$ & Share & $\begin{array}{c}\% \\
\text { share }\end{array}$ \\
\hline Residence in urban & $\begin{array}{c}0.011 \\
0\end{array}$ & $\begin{array}{c}0.31 \\
82\end{array}$ & 0.0038 & $\begin{array}{c}0.566 \\
1\end{array}$ & 0.0021 & 6 \\
\hline
\end{tabular}

Mother's education (No education ${ }^{\mathrm{a}}$ )

\begin{tabular}{|c|c|c|c|c|c|c|}
\hline Incomplete primary* & $\begin{array}{c}0.025 \\
4 \\
\end{array}$ & $\begin{array}{c}0.04 \\
86 \\
\end{array}$ & 0.0013 & $\begin{array}{c}- \\
0.260 \\
6 \\
\end{array}$ & $\begin{array}{c}- \\
0.0003 \\
\end{array}$ & -1 \\
\hline Complete primary* & $\begin{array}{c}0.041 \\
4 \\
\end{array}$ & $\begin{array}{c}0.03 \\
85 \\
\end{array}$ & 0.0017 & $\begin{array}{c}- \\
0.117 \\
7\end{array}$ & $\begin{array}{c}- \\
0.0002 \\
\end{array}$ & -1 \\
\hline Incomplete secondary* & $\begin{array}{c}0.046 \\
0 \\
\end{array}$ & $\begin{array}{c}0.14 \\
37 \\
\end{array}$ & 0.0072 & $\begin{array}{c}- \\
0.097 \\
7 \\
\end{array}$ & $\begin{array}{c}- \\
0.0007 \\
\end{array}$ & -2 \\
\hline Complete secondary* & $\begin{array}{c}0.063 \\
7\end{array}$ & $\begin{array}{c}0.43 \\
03 \\
\end{array}$ & 0.0298 & $\begin{array}{c}0.073 \\
9 \\
\end{array}$ & 0.0022 & 6 \\
\hline Higher* & $\begin{array}{c}0.119 \\
6 \\
\end{array}$ & $\begin{array}{c}0.16 \\
08 \\
\end{array}$ & 0.0209 & $\begin{array}{c}0.413 \\
4 \\
\end{array}$ & 0.0086 & 25 \\
\hline Sum & & & & & & 28 \\
\hline
\end{tabular}

Wealth index (Poorest ${ }^{\mathrm{a}}$ )

\begin{tabular}{|l|c|c|c|c|c|c|}
\hline & 0.006 & 0.19 & & - & & \\
Poorer & 0 & 33 & 0.0013 & 8 & 0.0006 & -2 \\
\hline & & & & - & & \\
Middle* & 0.027 & 0.24 & & 0.021 & - & \\
\hline & 7 & 85 & 0.0075 & 0 & 0.0002 & 0 \\
Richer* & 0.064 & 0.21 & & 0.442 & & \\
\hline & 3 & 47 & 0.0150 & 2 & 0.0066 & 19 \\
Richest* & 0.128 & 0.17 & & 0.828 & & \\
\hline Sum & 5 & 15 & 0.0239 & 5 & 0.0198 & 57 \\
\hline
\end{tabular}

Mother's age $\left(15-24^{\mathrm{a}}\right)$

\begin{tabular}{|l|c|c|c|c|c|c|}
\hline & 0.001 & 0.70 & & 0.021 & 0.0000 & \\
$25-39$ & 2 & 71 & 0.0009 & 6 & 2 & 0 \\
\hline & & & & - & - & \\
$40-49$ & 0.009 & 0.04 & & 0.057 & 0.0000 & \\
\hline
\end{tabular}




\begin{tabular}{|c|c|c|c|c|c|c|}
\hline decomposing the socioeconomic & \multicolumn{3}{|c|}{ Mohamed ali Hussein } & \multicolumn{3}{|c|}{ publishing date $24 / 9 / 2020$} \\
\hline Sum & & & & & & 0 \\
\hline \multicolumn{7}{|c|}{ Partner's education (No education ${ }^{a}$ ) } \\
\hline Incomplete primary & $\begin{array}{c}0.010 \\
7 \\
\end{array}$ & $\begin{array}{c}0.07 \\
71 \\
\end{array}$ & 0.0009 & $\begin{array}{c}- \\
0.183 \\
7 \\
\end{array}$ & $\begin{array}{c}- \\
0.0002 \\
\end{array}$ & 0 \\
\hline Complete primary* & $\begin{array}{c}0.026 \\
1\end{array}$ & $\begin{array}{c}0.05 \\
70\end{array}$ & 0.0016 & $\begin{array}{c}0.056 \\
2 \\
\end{array}$ & $\begin{array}{c}- \\
0.0000 \\
9\end{array}$ & 0 \\
\hline Incomplete secondary & $\begin{array}{c}0.005 \\
4\end{array}$ & $\begin{array}{c}0.12 \\
42\end{array}$ & 0.0007 & $\begin{array}{c}- \\
0.091 \\
3\end{array}$ & $\begin{array}{c}- \\
0.0000 \\
7\end{array}$ & 0 \\
\hline Complete secondary & $\begin{array}{c}0.009 \\
8\end{array}$ & $\begin{array}{c}0.44 \\
28\end{array}$ & 0.0047 & $\begin{array}{c}0.014 \\
3 \\
\end{array}$ & $\begin{array}{c}0.0000 \\
8 \\
\end{array}$ & 0 \\
\hline Higher & $\begin{array}{c}0.020 \\
5\end{array}$ & $\begin{array}{c}0.17 \\
02\end{array}$ & 0.0038 & $\begin{array}{c}0.339 \\
6\end{array}$ & 0.0013 & 4 \\
\hline Sum & & & & & & 3 \\
\hline \multicolumn{7}{|l|}{$\begin{array}{l}\text { Region (Urban } \\
\text { governorates a) }\end{array}$} \\
\hline Urban Lower Egypt & $\begin{array}{c}0.019 \\
2 \\
\end{array}$ & $\begin{array}{c}0.09 \\
40 \\
\end{array}$ & 0.0020 & $\begin{array}{c}0.581 \\
2 \\
\end{array}$ & 0.0011 & 3 \\
\hline Rural Lower Egypt & $\begin{array}{c}0.021 \\
5 \\
\end{array}$ & $\begin{array}{c}0.38 \\
99 \\
\end{array}$ & 0.0091 & $\begin{array}{c}0.149 \\
2 \\
\end{array}$ & $\begin{array}{c}- \\
0.0014 \\
\end{array}$ & -4 \\
\hline Urban Upper Egypt & $\begin{array}{c}- \\
0.022 \\
0\end{array}$ & $\begin{array}{c}0.11 \\
09\end{array}$ & -0.0027 & $\begin{array}{c}0.456 \\
0 \\
\end{array}$ & $\begin{array}{c}- \\
0.0012 \\
\end{array}$ & -3 \\
\hline Rural Upper Egypt & $\begin{array}{c}- \\
0.022 \\
7 \\
\end{array}$ & $\begin{array}{c}0.28 \\
77\end{array}$ & -0.0071 & $\begin{array}{c}- \\
0.418 \\
5\end{array}$ & 0.0030 & 9 \\
\hline Frontier governorates $*$ & $\begin{array}{c}- \\
0.045 \\
8 \\
\end{array}$ & $\begin{array}{c}0.00 \\
94\end{array}$ & -0.0005 & $\begin{array}{c}0.105 \\
8 \\
\end{array}$ & $\begin{array}{c}- \\
0.0000 \\
5\end{array}$ & 0 \\
\hline Sum & & & & & & 4 \\
\hline Working mother & $\begin{array}{c}- \\
0.010 \\
2 \\
\end{array}$ & $\begin{array}{c}0.11 \\
42 \\
\end{array}$ & -0.0013 & $\begin{array}{c}0.221 \\
5 \\
\end{array}$ & $\begin{array}{c}- \\
0.0003 \\
\end{array}$ & -1 \\
\hline $\begin{array}{l}\text { Having a terminated } \\
\text { pregnancy }\end{array}$ & $\begin{array}{c}0.006 \\
9 \\
\end{array}$ & $\begin{array}{c}0.20 \\
61 \\
\end{array}$ & 0.0016 & $\begin{array}{c}- \\
0.065 \\
4\end{array}$ & $\begin{array}{c}- \\
0.0001\end{array}$ & 0 \\
\hline Reading Newspaper & $\begin{array}{c}0.015 \\
1\end{array}$ & $\begin{array}{c}0.13 \\
92\end{array}$ & 0.0023 & $\begin{array}{c}0.284 \\
7\end{array}$ & 0.0006 & 2 \\
\hline Listening to Radio & $\begin{array}{c}- \\
0.000 \\
1\end{array}$ & $\begin{array}{c}0.20 \\
95\end{array}$ & $\begin{array}{c}0.0000 \\
2\end{array}$ & $\begin{array}{c}0.085 \\
4\end{array}$ & $\begin{array}{c}- \\
0.0000 \\
\end{array}$ & 0 \\
\hline
\end{tabular}




\begin{tabular}{|c|c|c|c|c|c|c|}
\hline decomposing the socioeconomic & \multicolumn{3}{|c|}{ Mohamed ali Hussein } & \multicolumn{3}{|c|}{ publishing date $24 / 9 / 2020$} \\
\hline Watching TV & $\begin{array}{c}0.023 \\
6\end{array}$ & $\begin{array}{c}0.98 \\
51\end{array}$ & $\begin{array}{c}0.0252 \\
63\end{array}$ & $\begin{array}{c}0.005 \\
4\end{array}$ & 0.0001 & 0 \\
\hline Total predicted C & & & & & 0.0402 & 116 \\
\hline Residual & & & & & $\begin{array}{c}- \\
0.0056\end{array}$ & -16 \\
\hline $\begin{array}{l}\text { Total C of skilled birth } \\
\text { assistance }\end{array}$ & & & & & $\begin{array}{c}0.0345 \\
8\end{array}$ & 100 \\
\hline
\end{tabular}

${ }^{a}$ Reference category * Significant at $1 \%$

Moreover, education of women is another significant determinant explaining a considerable contribution of the inequalities in skilled birth assistance. Our findings demonstrate that the share of women's education to inequality in skilled assistance during childbirth was 28 which illustrated if education was equally distributed among different mothers, then inequality in skilled birth assistance would have been by 28 percent lower. Furthermore, the findings from Table [3] clearly show that residence in urban areas $(6 \%)$, region $(4 \%)$, and partner's education $(3 \%)$ showed remarkable shares to inequality in skilled birth assistance.

\section{Discussion}

Our findings show that pro-rich inequality existed in indicators of delivery care in Egypt. Moreover, the two concentration indices of delivery care were positive which indicated that mothers from lower socioeconomic groups are less likely to use delivery services. These findings were like many others in delivery care studies in various pieces of the world [Houweling et al. 2007; Zere et al.2011; Liu et al. 2014].

Our analysis indicates that wealth index was demonstrated to be the greatest share to the inequality in use of delivery services. These results agree with the findings from similar researches in different countries (Say and Raine 2007; Ahmed et al. 2010; Zere 
et al.2011; Liu et al. 2014; Paredes K. P., 2016; Goli. et al 2017; Makate and Makate 2017; Tesfaye et al.2017;).

It can be concluded that education of women increases understanding of the importance of health issues, which thus prompts to greater utilization of delivery services (Greenaway et al. 2012). Woman's education is considered as a significant predictor of use of delivery services. The analysis in this paper revealed that mother's education can clarify a large contribution of the inequalities in utilization of delivery care services. This agrees with results from previous studies from Nepal and Bangladesh (Goli et al 2017) and other parts of the world (Paredes K. P., 2016; Huda et al 2018). However, a previous study in Zimbabwe [Makate and Makate 2017] found that education can clarify a reasonable contribution of the inequalities in delivery services utilization.

Our findings demonstrate that the share of wealth index to the inequality in use of delivery services was more than the contribution of women's education. This result from the decomposition analysis of inequality is in conformity with what is demonstrated in several previous researches in Bangladesh, Pakistan, Nepal and Philippines [Huda et al 2018; Paredes K. P., 2016]. While as noted in a previous research in Namibia [Zere et al 2011], the share of mother's education to the inequality in utilization of delivery health services was more than the contribution of wealth index.

\section{Conclusion}

This study clarified inequality in utilization of delivery care indicators and decomposed inequality into its determinants in Egypt using data from EDHS 2014. The current paper carried out decomposition analyses to measure the relative share of 
demographic and socioeconomic determinants to disparities in use of delivery services.

The findings of the decomposition method showed that prorich inequality existed in use of delivery care indicators in Egypt. The results of the current paper clearly indicate that the highest share of the total inequality in use of delivery care in Egypt was wealth index, followed by maternal education.

In conclusion, our results of the decomposition analyses suggest that eradication disparities in use of delivery services may be achieved by equitable access to reproductive health services from both poor and rich groups. Moreover, governmental policies in Egypt must be directed towards illiterate and poor women to increase awareness about the importance of institutional delivery and the risks of home delivery. Greater emphasis on maternal education would increase access to delivery services through improved knowledge and attitudes [Goli 2017; Zere et al 2011].

One of the limitations of the current paper is that the decomposition analyses could not include many other important determinants of inequalities in usage of delivery services such as the distance to health facility delivery, complications during pregnancy, quality and availability of health services. Some of previous studies found that complications during pregnancy are important determinants to the inequalities in delivery care utilization [Arba et al. 2016; Bayu et al. 2015; Kamal et al. 2015]. However, Huda (2018) reported that complications during pregnancy contributed minimally towards the use of delivery services [Huda et al 2018]. Future research may include such these determinants of institutional delivery to measure the relative contribution of these determinants in Egypt. 


\section{References}

[1] Ahmed S, Creanga AA, Gillespie DG and Tsui AO (2010).

Economic status, education and empowerment: implications for maternal health service utilization in developing countries. PLoS One, 5(6).

[2] Arba MA, Darebo TD and Koyira MM (2016). Institutional Delivery Service Utilization among Women from Rural Districts of Wolaita and Dawro Zones, Southern Ethiopia; a Community Based Cross-Sectional Study. PLoS ONE 11(3): e0151082. doi:10.1371/journal.pone.0151082

[3] Bayu H, Adefris M, Amano A and Abuhay M (2015). Pregnant women's preference and factors associated with institutional delivery service utilization in Debra Markos Town, North West Ethiopia: a community based follow up study. BMC Pregnancy and Childbirth, 15(15). Medline:25652361 doi:10.1186/s12884015-0437-z

[4] Bobo FT, Yesuf EA and Woldie M (2017). Inequities in utilization of reproductive and maternal health services in Ethiopia. International Journal for Equity in Health, 16:105. DOI 10.1186/s12939-017-0602-2.

[5] Goli S, Nawal D, Rammohan A, Sekher TV, and Singh D (2017). Decomposing the socioeconomic inequality in utilization of maternal health care services in selected countries of south Asia and sub-Saharan Africa. Journal of Biosocial Science, 1-21.

[6] Greenaway ES, Leon J, Baker DP. (2012). Understanding the Association between maternal education and use of health services in Ghana: Exploring the role of health knowledge. J Biosoc Sci., 44(6):733-47. 
[7] Houweling TA, Kunst AE, Huisman M, Mackenbach JP (2007). Using relative and absolute measures for monitoring health inequalities: experiences from cross-national analyses on maternal and child health. International Journal for Equity in Health, 6:15.

[8] Huda T M, Hayes A and Dibley M J (2018). Examining horizontal inequity and social determinants of inequality in facility delivery services in three South Asian countries. Journal of global health, 8(1).

[9] Hussein MA (2018). Socioeconomic Inequality in Delivery Care: Evidence from a Cross-Sectional Study in Egypt. Institute of Statistical Studies and Research, Cairo University, Egypt.

[10] Kamal SM, Hassan CH and Alam GM (2015). Determinants of institutional delivery among women in Bangladesh. Asia Pac J Public Health, 27(2):1372-88. Medline:23666835 doi: $10.1177 / 1010539513486178$

[11] Khadr Z (2009). Monitoring socioeconomic inequity in maternal health indicators in Egypt: 1995-2005. International Journal for Equity in Health, 8:38. Doi:10.1186/1475-9276-8-38

[12] Liu X, Gao W and Yan H (2014). Measuring and decomposing the inequality of maternal health services utilization in Western Rural China. BMC Health Services Research, 14:102.

[13] Makate $M$ and Makate $C$ (2017). The evolution of socioeconomic status related inequalities in maternal health care utilization: evidence from Zimbabwe, 1994-2011. Global Health Research and Policy, 2:1

[14] Paredes KP (2016). Inequality in the use of maternal and child health services in the Philippines: do pro-poor health policies result in more equitable use of services. International Journal for Equity in Health, 15:181. 
[15] Say L and Raine R (2007). A systematic review of inequalities in the use of maternal health care in developing countries: examining the scale of the problem and the importance of context. Bulletin of the World Health Organization, 85(10).

[16] Tesfaye B, Mathewos T, and Kebede M (2017). Skilled delivery inequality in Ethiopia: to what extent are the poorest and uneducated mothers benefiting. International Journal for Equity in Health, 16:82.

[17] Wagstaff A, Paci P and Doorslaer EV (1991). On the measurement of inequalities in health. Social Science and Medicine, $33,545-557$.

[18] Wagstaff A, Kakwani NC and Doorlsaer EV (1997). Socioeconomic inequalities in health: Measurement, computation and statistical inference. Journal of Econometrics, 77(1), 87-104.

[19] Wagstaff A, van Doorslaer E and Watanabe N (2003). On decomposing the causes of health sector inequalities with an application to malnutrition inequalities in Vietnam. $\mathrm{J}$ Econom, 112:207-23.

[20] World Health Organization (2005). Make Every Mother and Child Count. Geneva: WHO.

[21] World Health Organization (2014). WHO Recommendations on Postnatal Care of the Mother and Newborn 2013. Geneva: WHO.

[22] World Health Organization (2019). Trends in maternal mortality 2000 to 2017: estimates by WHO, UNICEF, UNFPA, World Bank Group and the United Nations Population Division. Geneva: WHO; 2019. Licence: CC BY-NC-SA 3.0 IGO.

[23] Zere E, Oluwole1 D, Kirigia JM, Mwikisa CN and Mbeeli T (2011): Inequities in skilled attendance at birth in Namibia: A decomposition analysis. BMC Pregnancy and Childbirth, 11:34. 